\title{
Hyper-Kamiokande Project
}

\section{Yury Kudenko*}

Institute for Nuclear Research of the Russian Academy of Sciences

117312 Moscow, Russia

E-mail: kudenko@inr.ru

Hyper-Kamiokande, a third generation 260 kt Water Cherenkov detector, will serve as a far neutrino detector of the Tokai-to-Hyper-Kamiokande (T2HK) experiment with a high intensity neutrino beam from J-PARC. The main goals of this project include a sensitive measurement of CP violation in neutrino oscillations, a search for proton decay and study of solar, atmospherics and astrophysical neutrinos.

Neutrino Oscillation Workshop (NOW2018)

9 - 16 September, 2018

Rosa Marina (Ostuni, Brindisi, Italy)

* Speaker. 


\section{Introduction}

The huge 260 kt Water Cherenkov detector, Hyper-Kamiokande, is being developed by an international collaboration as a leading worldwide experiment to address fundamental unsolved questions in particle physics and cosmology [1,2]. It will be used as a far neutrino detector in the long baseline experiment T2HK which will use the neutrino beam from the upgraded Japan Proton Accelerator Research Complex (J-PARC). Hyper-Kamiokande will be able to measure with highest sensitivity the leptonic $\mathrm{CP}$ violation. It has an excellent capability to search for proton decay and strong astrophysical program.

\section{Hyper-Kamiokande detector}

The schematic view of the baseline configuration of Hyper-Kamiokande is shown in Fig. 1. The design is a cylindrical tank with a diameter of $74 \mathrm{~m}$ and height of $60 \mathrm{~m}$. The total (fiducial)

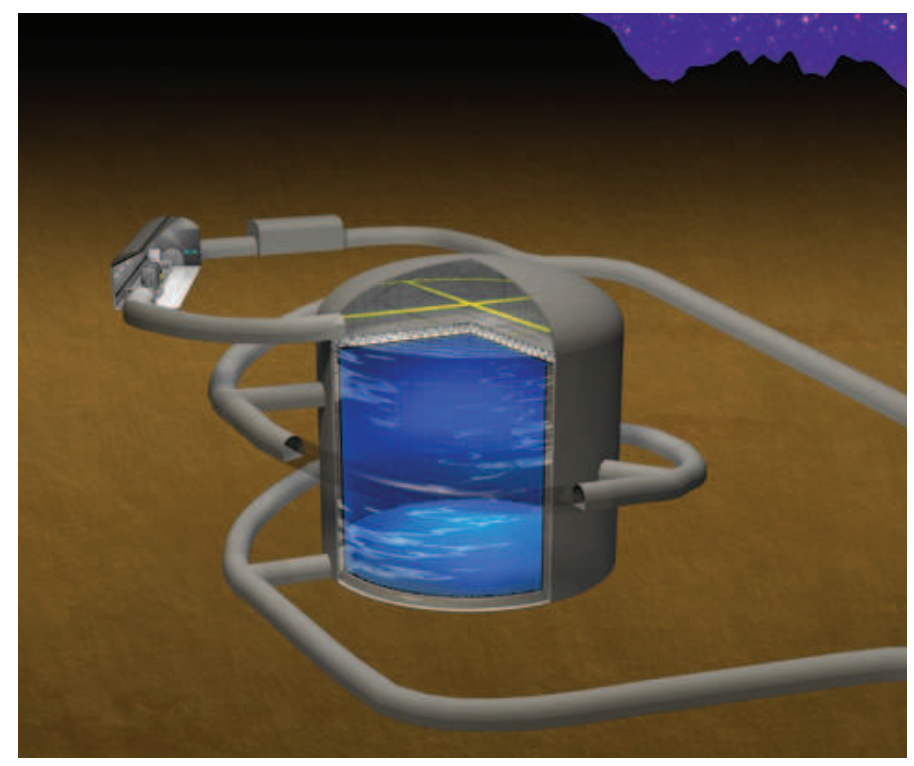

Figure 1: Schematic view of the Hyper-Kamiokande tank.

mass of the detector is 258 (187) kt, giving a fiducial mass that is 8 times larger than SuperKamiokande. The Hyper-Kamiokande detector will be located in Tochibora mine, $8 \mathrm{~km}$ south of Super-Kamiokande and $295 \mathrm{~km}$ away from J-PARC. For an overburden of $650 \mathrm{~m}$ of rock or 1,750 meters-water-equivalent the muon flux is about $7.5 \times 10^{-7} \mathrm{~cm}^{-2} s^{-1}$, i.e. about 5 times higher than in Super-Kamiokande. The detector is filled with highly transparent purified water which has a light attenuation length of $\sim 100 \mathrm{~m}$. Hyper-Kamiokande will be instrumented with inward facing newly developed high-efficiency $50 \mathrm{~cm}$ diameter Hamamatsu R12860 PMTs which have $\sim 2$ times higher photon detection efficiency than that of the Super-Kamiokande PMTs. The inner detector region of the tank is viewed by 40,000 PMTs, providing $40 \%$ photo-cathode coverage, equivalent to that of Super-Kamiokande. Hyper-Kamiokande will use the water Cherenkov ring-imaging technique to detect charged leptons produced in neutrino interactions on nuclei in water. The number of photons and their arrival times on the photodetectors are used to reconstruct the energy and the vertex of the 
particle, respectively. At these energies, most neutrino-nucleus interactions are quasi-elastic, and the measurement of the outgoing charged lepton allows us to obtain an accurate reconstruction of the energy and flavor of the initial neutrino.

\section{Physics potential}

\subsection{CP violation}

The long-baseline neutrino experiment T2HK will use an intense high quality $2.5^{\circ}$ off-axis neutrino beam from the $30 \mathrm{GeV}$ proton Synchrotron at J-PARC, a near/intermediate detector complex and Hyper-Kamiokande as a far detector. The quasi-monoenergetic beam comprises mostly of $v_{\mu}\left(\bar{v}_{\mu}\right)$ with the peak energy of $600 \mathrm{MeV}$ tuned to the first oscillation maximum at $295 \mathrm{~km}$. The J-PARC beam will be upgraded to the beam power of $1.3 \mathrm{MW}$. To reduce the systematic uncertainties on the total event prediction in the far detector, in presence of oscillation, to $\leq 4 \%$ the ND280 near detector will be upgraded [3] before the start of Hyper-Kamiokande. The existing $\pi^{0}$ detector will be replaced by a new horizontal tracking system surrounded by a time-of-flight detector. An intermediate water Cherenkov detector [4] is proposed to be constructed at a distance of 1-2 km from the pion-production target. It will measure the neutrino cross section on water with the same solid angle as the far detector. A combination of the magnetized ND280 detector and the water Cherenkov detector will allow to further reduce systematic errors.

For direct measurement of $\mathrm{CP}$ asymmetry in neutrino oscillations, the comparison between the oscillation probabilities of $v_{\mu} \rightarrow v_{e}$ and $\bar{v}_{\mu} \rightarrow \bar{v}_{e}$ is necessary. The information about CP phase $\delta_{C P}$ can be obtained from the total number the energy spectrum of the detected $v_{e}$ and $\bar{v}_{e}$ events. For $E_{v} \sim 0.6 \mathrm{GeV}$, the baseline of $295 \mathrm{~km}, \sin ^{2} 2 \theta_{13}=0.1, \sin ^{2} 2 \theta_{23}=1.0$ the CP asymmetry

$$
A_{C P}=\frac{P\left(v_{\mu} \rightarrow v_{e}\right)-P\left(\bar{v}_{\mu} \rightarrow \bar{v}_{e}\right)}{P\left(v_{\mu} \rightarrow v_{e}\right)+P\left(\bar{v}_{\mu} \rightarrow \bar{v}_{e}\right)} \simeq-0.29 \sin \delta_{C P}+0.09
$$

The effect of the CP violating term can be as large as $29 \%$, while that of the matter effect is $9 \%$. For study of the sensitivity to $\mathrm{CP}$ violation, an integrated beam power $1.3 \mathrm{MW} \times 10^{8} \mathrm{sec}$ that corresponds to $2.7 \times 10^{22}$ protons on target (POT) with the $30 \mathrm{GeV} \mathrm{J-PARC} \mathrm{proton} \mathrm{beam} \mathrm{is}$ assumed. The ratio of integrated beam power for neutrino and antineutrino beam mode is fixed $v: \bar{v}=1: 3$. This allows Hyper-Kamiokande to detect about the same number of electron neutrino and antineutrino events. The selection criteria of $v_{e}$ and $v_{\mu}$ candidate events are based on those established in the Super-Kamiokande and T2K experiments. The total systematic uncertainties of the number of expected events are assumed to be $3.2 \%$ for the $v_{e}$ appearance and $3.9 \%$ for the $\bar{v}_{e}$ appearance. Fig. 2 shows the expected significance to exclude $\delta_{C P}=0$ or $\pi$ (the CP conserving cases) after 10 years of data taking. As seen from Fig. 2, CP violation in neutrino oscillations can be observed with $\geq 5(3) \sigma$ significance for $57(80) \%$ of the possible values of $\delta_{C P}$. Exclusion of $\delta_{C P}=0$ can be obtained with a significance of $8 \sigma$ in the case of maximal CP violation with $\delta_{C P}=-\pi / 2$.

An interesting approach to build the second identical detector in Korea at a distance of $1000-$ $1300 \mathrm{~km}$ from J-PARC is also considered [5]. This second far detector can be exposed by 1-3 degree off-axis neutrino beams from J-PARC. This two-baseline experiment can measure oscillations at 


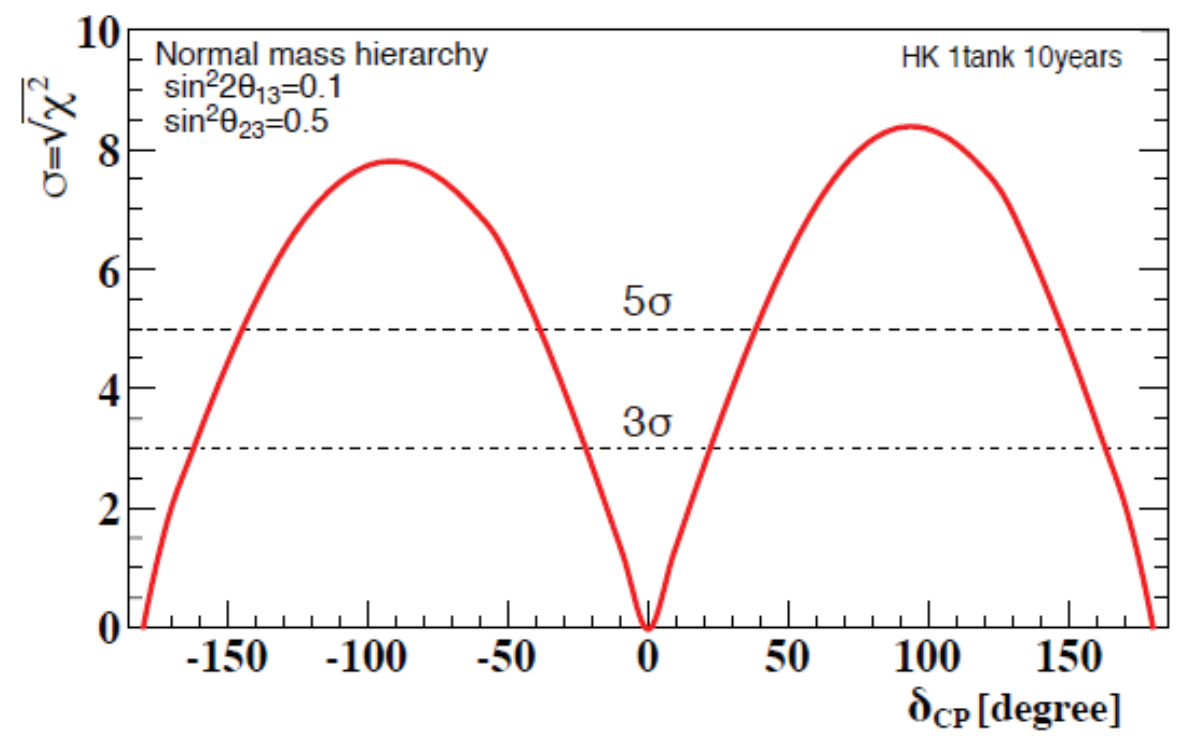

Figure 2: Expected significance to exclude CP conservation $\left(\delta_{C P}=0\right.$ or $\pi$ ) for the normal mass order. The significance is calculated as $\sqrt{\Delta \chi^{2}}$, where $\Delta \chi^{2}$ the difference of $\chi^{2}$ for the trial value $\delta_{C P}$ and for $\delta_{C P}=0$ or $\pi$. The smaller value of difference is taken.

both the first and second oscillation maxima that allows the experiment to break the degeneracy of oscillation parameters and to increase the sensitivity to oscillation physics.

\subsection{Proton decay}

Grand Unified theories (GUTs) are well motivated theoretical concepts beyond the Standard Model. They unify the strong, weak and electromagnetic forces into a single force. In these models, quarks and leptons are treated similarly and baryon number is violated because leptons can interact with quarks. The general feature of GUTs is the prediction of the instability of protons by baryon number violating decays.

The decay $p \rightarrow e^{+} \pi^{0}$ is the favorite mode in many GUTs. In one of the simplest model SU(5), the proton decay is mediated by the massive $X$ and $Y$ gauge boson exchange. The lifetime of the proton is given by

$$
\tau\left(p \rightarrow e^{+} \pi^{0}\right) \sim \frac{M_{G}^{4}}{m_{p}^{5}}
$$

where $m_{p}$ is the mass of the proton and the $X$ and $Y$ masses are denoted as $M_{G}$. Cherenkov detectors are very suitable for a search for the decay $p \rightarrow e^{+} \pi^{0}$ because it has a very clean event topology with no invisible particles in the final state. In this decay the proton mass is converted into three electromagnetic showers corresponding to positron and two photons from $\pi^{0} \rightarrow \gamma \gamma$ decay, with a small total momentum. This channel has a very clear signature in Hyper-Kamiokande. For events with three rings which have two rings with invariant mass $m_{\gamma \gamma}$ close to the $\pi^{0}$ mass, a cut $85<m_{\gamma \gamma}<185 \mathrm{MeV}$ is applied. The signal is selected if its total mass is in the range 800$1050 \mathrm{MeV}$ and the total momentum $p_{t o t}$ is less than $250 \mathrm{MeV} / \mathrm{c}$. The proton decay candidates divided into two signal regions: a free proton decay region with $p_{t o t}<100 \mathrm{MeV} / \mathrm{c}$ and a bound proton decay region $100<p_{\text {tot }}<250 \mathrm{MeV} / \mathrm{c}$. Atmospheric neutrino interactions give the main 
contribution to the background in proton decay searches. Fig. 3 shows the expected distribution

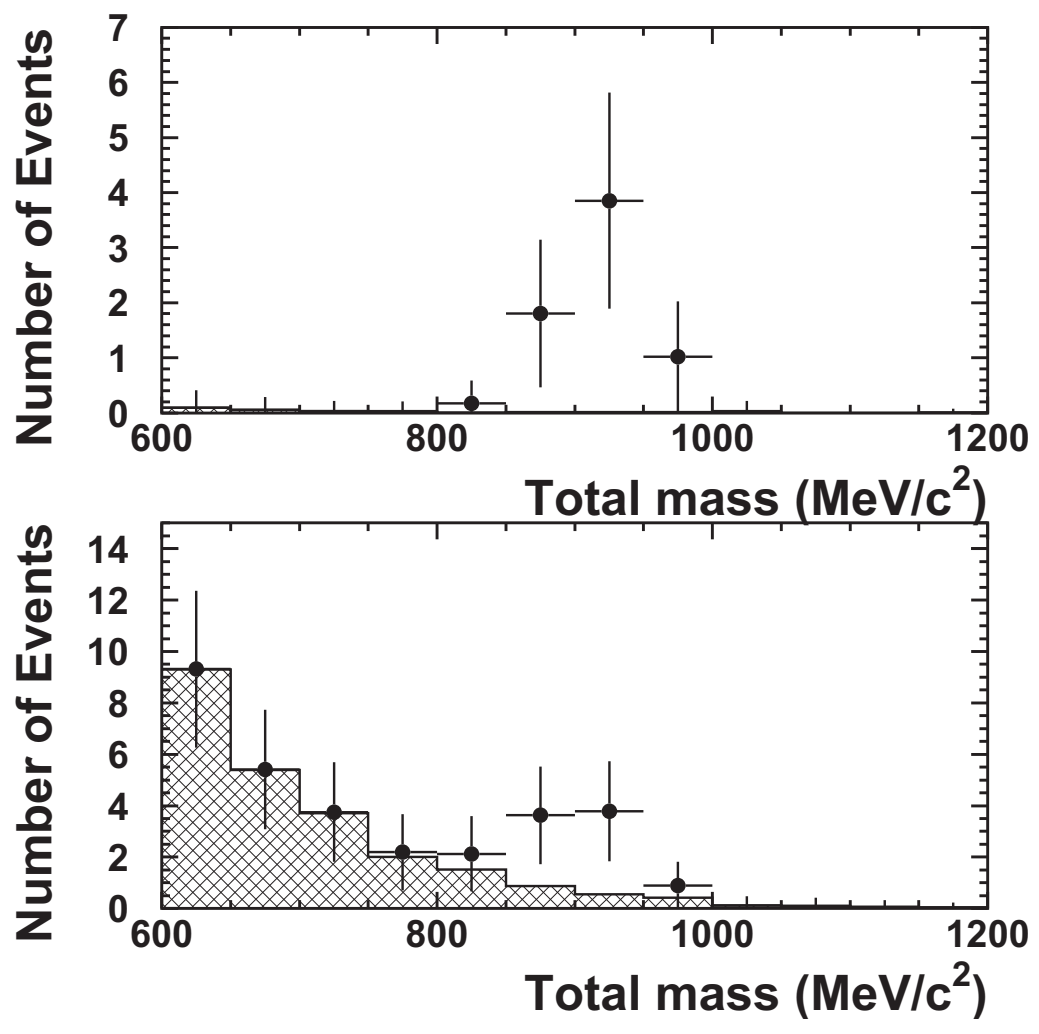

Figure 3: Reconstructed invariant mass distribution of events passed all cuts applied for search of the $p \rightarrow$ $e^{+} \pi^{0}$ decay after 10 years of exposure of Hyper-Kamiokande. The hatched histograms show the atmospheric neutrino background. Solid crosses show the sum of the proton decay signal for the lifetime of $1.7 \times 10^{34}$ years and background. The upper panel corresponds to the free proton decay, the lower panel shows the bound proton decay.

of the proton invariant mass for signal events and atmospheric neutrino background assuming the proton lifetime of $1.7 \times 10^{34}$ years (just beyond the Super-Kamiokande current limit) after 10 years of exposure. The notable difference in the signal-to-background ratio between the free and bound proton is seen. The signal efficiency of free proton decays is about $87 \%$, however the nuclear effects reduce the efficiency for bound proton decays such the overall efficiency for all decays is reduced to $\sim 40 \%$. Based on these numbers the $90 \%$ CL sensitivity of Hyper-Kamiokande is expected to be $\sim 8 \times 10^{34}$ years for a 10-year exposure. For the $p \rightarrow \bar{v} K^{+}$mode, the $90 \% \mathrm{CL}$ limit of $3 \times 10^{34}$ years is expected to be obtained. Hyper-Kamiokande is sensitive to many other nucleon decay modes, as described in Ref. [1].

\subsection{Atmospheric neutrinos}

Atmospheric neutrinos will be used by Hyper-Kamiokande to study the neutrino mass hierarchy. The excellent ability of this detector to distinguish the charge current $v_{\mu}$ and $v_{e}$ interactions allows the detector to test the mass hierarchy in both $v_{\mu} \rightarrow v_{\mu}$ and $v_{\mu} \rightarrow v_{e}$ channels. Matter induced resonant-like oscillations in the energy range of $2-10 \mathrm{GeV}$ significantly enhance either the 
$v_{\mu} \rightarrow v_{e}$ or the $\bar{v}_{\mu} \rightarrow \bar{v}_{e}$ probability for upward-going neutrinos depending on the mass hierarchy. The ten-year expected sensitivity to resolve the mass hierarchy for the 260 kton Hyper-Kamiokande detector is shown in Fig. 4. This strong dependence of the sensitivity to hierarchy on $\theta_{23}$ is a typical

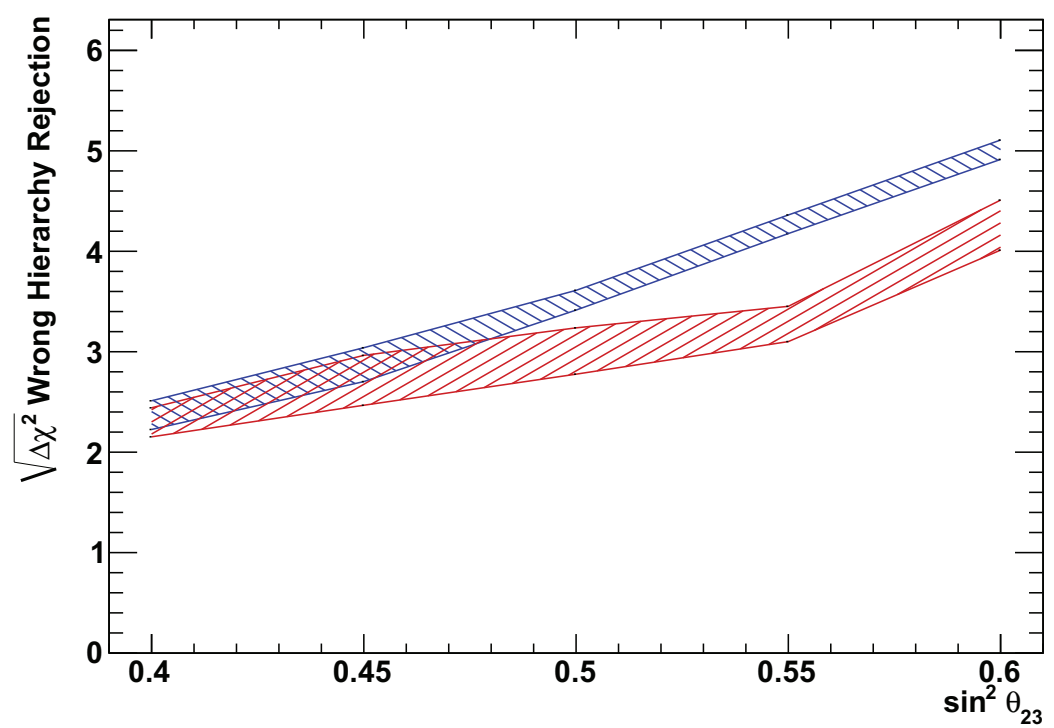

Figure 4: Mass hierarchy as a function of the true value of $\sin ^{2} 2 \theta_{23}$ at a fiducial exposure of $(187 \mathrm{kton}) \times$ (10 years) . The blue (red) band corresponds to the normal (inverted) hierarchy. The shaded bands show the uncertainty of $\delta_{C P}$.

feature of experiments with atmospheric neutrinos. The relatively short baseline of T2HK limits its sensitivity to the mass hierarchy, but provides a precise measurement of $\theta_{23}$. Combining the atmospheric and accelerator data Hyper-Kamiokande will be able to significantly improve the sensitivity to the mass hierarchy.

\subsection{Neutrino astrophysics}

Core-collapse supernova explosions are a copious source of all flavor neutrinos. The flavor composition, energy spectrum and time structure of the neutrino burst from a galactic supernova can provide the information about the explosion mechanism and the mechanisms of proto neutron star cooling. Hyper-Kamiokande primarily detects electron antineutrinos from the supernova explosion through the reaction $\bar{v}_{e}+p \rightarrow e^{+}+n$ with the detailed and time-dependent energy spectrum above the threshold of $\sim 3 \mathrm{MeV}$ while the DUNE experiment [6] which uses LAr TPC's will mostly detect electron neutrinos. Hyper-Kamiokande is expected to see $(50-80) \times 10^{3}$ events for a 10 kpc supernova and $(2-3) \times 10^{3}$ events for a supernova at the Large Magellanic Cloud where SN1987a was located.

Supernova relic neutrinos (SRN) produced by all supernova explosions since the beginning of the Universe have not yet been observed. Hyper-Kamiokande can measure SRN neutrinos in the energy range 16-30 MeV. Fig. 5 shows the expected SRN signal at the exposure of 10 years. It is estimated that 140 SRN events will be detected after 20 years of observation with the HyperKamiokande detector corresponding to a $5.2 \sigma$ statistical significance of the SRN signal. 


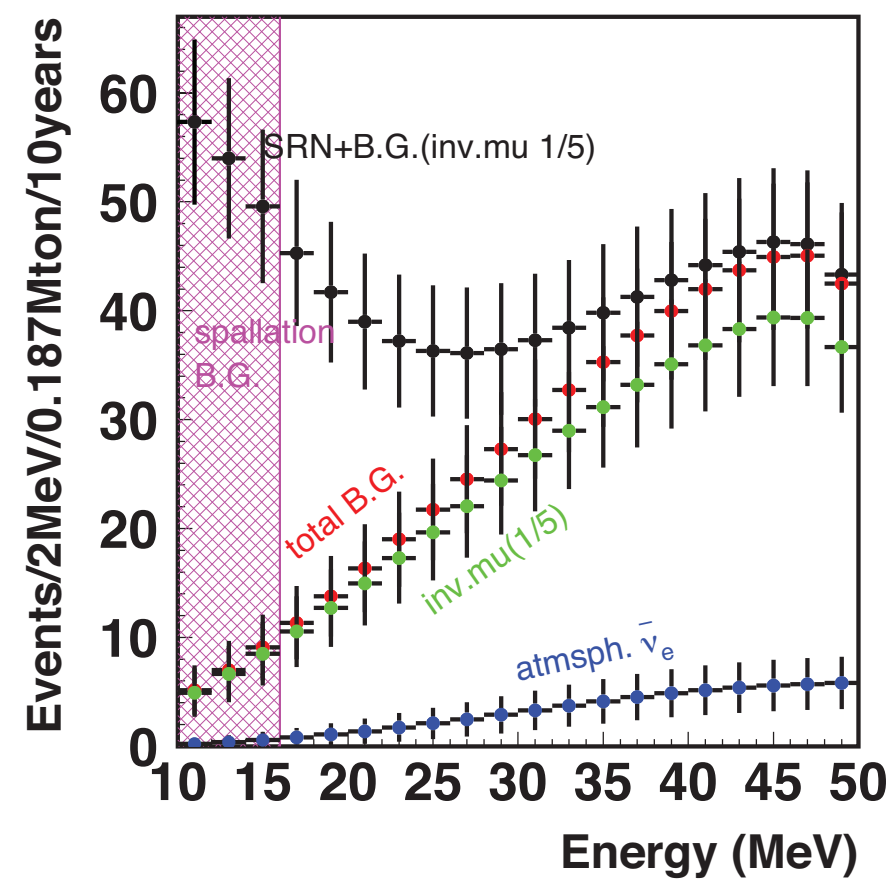

Figure 5: Expected spectrum of supernova relic neutrinos with 10 years of livetime with the neutron tagging efficiency of $67 \%$. The black dots show the sum of the signal and the total background [1].

\section{Conclusion}

Hyper-Kamiokande, the next generation large water Cherenkov detector, is being developed by an international collaboration. This multipurpose detector has a rich physics program in neutrino physics, astrophysics, and nucleon decay. The construction of the Hyper-Kamiokande detector is expected to start in 2020.

This work was supported in part by the RFBR/JSPS grant \# 17-52-50038.

\section{References}

[1] K.Abe et al., Hyper-Kamiokande Design Report, arXiv:1805.04163 [physics.ins-det].

[2] K.Abe et al., Physics potential of a long-baseline neutrino oscillation experiment using a J-PARC neutrino beam and Hyper-Kamiokande PTEP 2015, 053C02 2015 [hep-ex/1502.05199].

[3] K.Abe et al., Proposal for an Extended Run of T2K to $20 \times 10^{21}$ POT, arXiv:1609.04111 [hep-ex].

[4] S.Bhadra et al., Letter of Intent to Construct a nuPRISM Detector in the J-PARC Neutrino Beamline, arXiv:1412.3086 [physics.ins-det].

[5] K.Abe et al., Physics Potentials with the Second Hyper-Kamiokande Detector in Korea, arXiv:1611.06118 [hep-ex].

[6] R.Acciarri et al., Long-Baseline Neutrino Facility (LBNF) and Deep Underground Neutrino Experiment (DUNE) : Conceptual Design Report, Volume 2: The Physics Program for DUNE at $L B N F$, arXiv:1512.06148 [physics.ins-det]. 